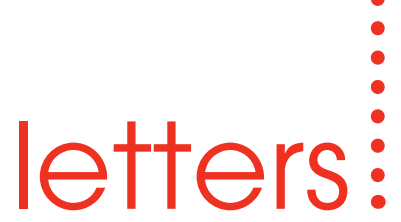

\title{
How many will lead to further intervention?
}

The article by David Bloom and Jay Padayachy, Vital guide to cosmetic dentistry (Vital summer 07 pages 15-22), raises a number of questions. The instance of endodontic retreatment (Figs 1 and 2) presumably includes the immediate post-operative radiograph since the periapical area is still present. The pulp chamber is empty. I could be more convinced of the success of the treatment if a follow-up radiograph showing resolution of the periapical area were shown, say a year after completion of the definitive coronal restoration.

Figures 5 and 6 show a number of heavily restored posterior teeth but it is wildly fanciful to imagine that these have failed. Most of them, particularly the premolars, look as if there would have been many years of service left. Any restoration could be described as 'failing' since all (including the mouthful of new composites) will fail eventually. A common reason for restoration replacement is secondary caries but no radiographic evidence of this was presented either before the wholesale destruction of the preoperative state or afterwards to show the quality of the work done. I wonder how many of those replacements will lead to further endodontic interventions which might not otherwise have been required.

I remain to be satisfied that the work carried out was necessary.

\section{Allan H. Cook}

Dental Surgeon

London

\section{Thank \\ you for your letter in response to our article in Vital.}

With regards to the re-root treatment we do have a follow-up radiograph taken 18 months later showing the area having healed and the pulp chamber filled with a fibre post and core. Please see radiograph.

The photographs showing the before and after pictures of quadrant replacement amalgams with direct and indirect restorations are an example of what can be achieved. In the molars there was marginal breakdown of the amalgams, a cuspal fracture that had been previously patched, the presence of ledges and failing interproximal contacts resulting in food impaction.

In the pre-molar region, the patient requested replacement of these shallow restorations on cosmetic grounds and these were replaced with direct composite under rubber dam using an incremental build up technique. In our experience the need for endodontics treatment, particularly in the heavily restored molars, is greatly reduced with restorations that are bonded to tooth tissue as these provide all the benefits of cuspal coverage (onlays and crowns) without the destruction of sound tooth tissue associated with a conventional crown preparation.

If you have any doubts we recommend you listen to Pascal Mange who is speaking for $B A C D$ in June 2008 on biomimetic restorative principles.

We trust this answers all your concerns. David Bloom and Jay Padayachy Authors of the article in question
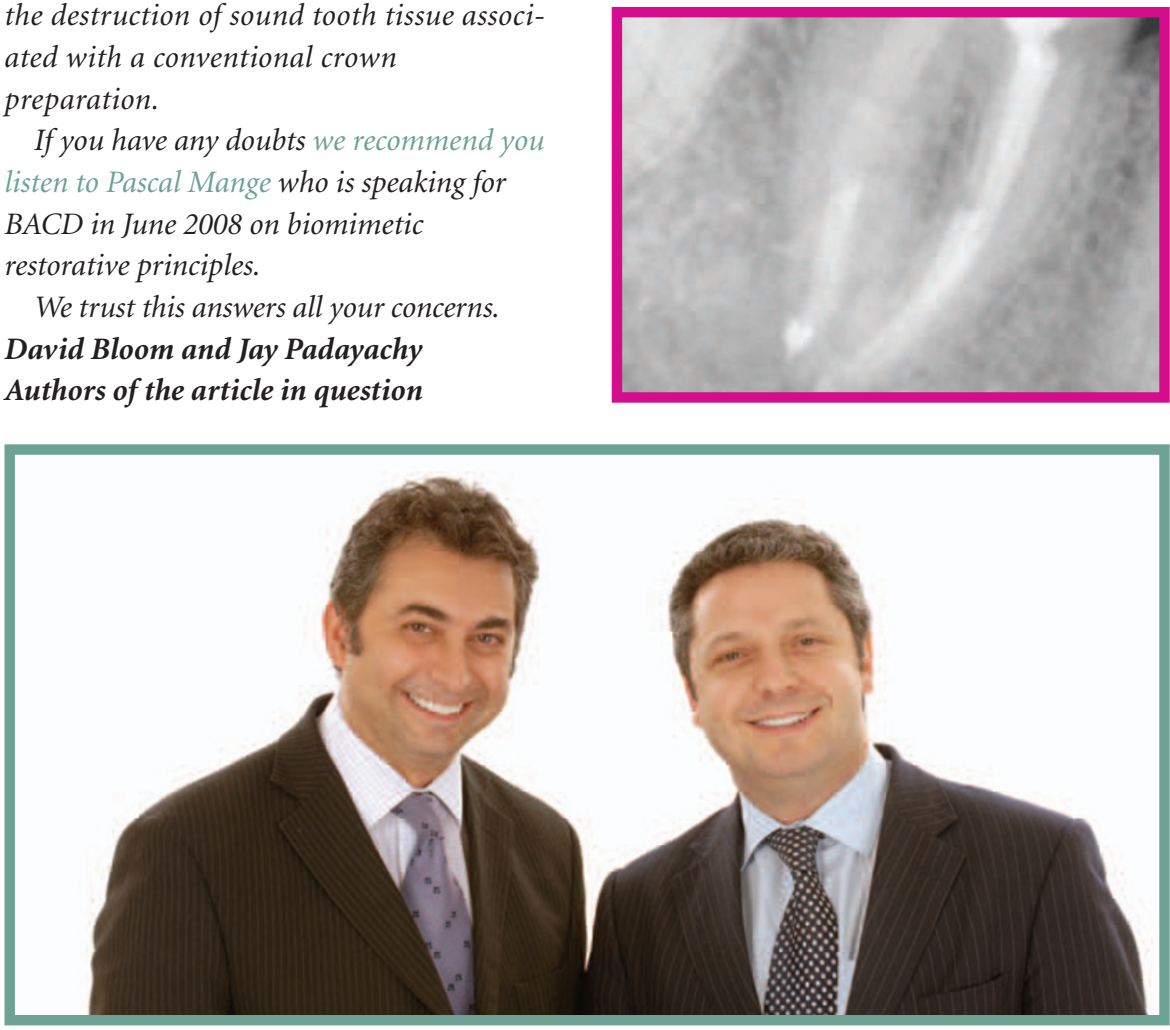

winter 0705 